\title{
FUNCTIONAL EQUATIONS ARISEN FROM THE CHARACTERIZATION OF BETA DISTRIBUTIONS
}

\author{
KÁROLY LAJKÓ AND FRUZSINA MÉSZÁROS
}

\begin{abstract}
Two functional equations, introduced by J. Wesołowski [8] related to an independence property for beta distributions, are investigated without any regularity conditions. The measurable solutions of equation (3) satisfied almost everywhere are also given.
\end{abstract}

\section{INTRODUCTION}

Let $X$ be a Beta-distributed random variable with parameters $p$ and $q$, where $p$ and $q$ are fixed positive numbers.

It is known that its density function is

$$
f(x)=\beta_{p, q}(x)=\left\{\begin{array}{lll}
\frac{1}{B(p, q)} x^{p-1}(1-x)^{q-1} & \text { if } & x \in(0,1) \\
0 & \text { if } & x \notin(0,1)
\end{array}\right.
$$

where

$$
B(p, q)=\int_{0}^{1} x^{p-1}(1-x)^{q-1} d x
$$

is the beta function.

Recently J. Wesołowski (see [8]) studied a new characterization of beta distribution by the transformation

$$
\psi:(0,1)^{2} \rightarrow(0,1)^{2}, \quad \psi(x, y)=\left(\frac{1-y}{1-x y}, 1-x y\right) .
$$

A possible characterization of univariate distributions is based on the following general Transformation Theorem.

Theorem 1. Let $X=\left(X_{1}, \ldots, X_{n}\right)$ be an absolutely continuous random variable with density function $f: \mathbb{R}^{n} \rightarrow \mathbb{R}$, which is zero outside of a region $\Omega_{x} \subset \mathbb{R}^{n}$. Let $\psi: \Omega_{x} \rightarrow \Omega_{y} \subset \mathbb{R}^{n}$ be a one-to-one transformation onto $\Omega_{y}$ and denote $\psi^{-1}$ its inverse transformation.

If the Jacobi determinant $J(y)=\operatorname{det}\left(\frac{\partial \psi^{-1}(y)}{\partial y}\right)$ exists, is continuous and does not change sign in $\Omega_{y}$, then the random variable $Y=\psi(X)$ is absolutely continuous with density function $g$ such that

$$
g(y)= \begin{cases}f\left(\psi^{-1}(y)\right)|J(y)| & \text { if } y \in \Omega_{y}\left(\text { or } y \in \Omega_{y} \text { a.e. }\right) \\ 0 & \text { if } y \in \mathbb{R}^{n} \backslash \Omega_{y} .\end{cases}
$$

2000 Mathematics Subject Classification. 39B40, 39B22, 60E05, 62E10

Key words and phrases. Beta distributions, characterizations of probability distributions, general solution, measurable solution a. e.

Research supported partly by OTKA, Grant No. NK 68040. 
The function $\psi$ defined in (1) is bijective, $\psi^{-1}=\psi$ and the Jacobi determinant of $\psi^{-1}$ is of the form

$$
J(u, v)=\frac{-v}{1-u v} \quad(u, v \in(0,1)) .
$$

It is easy to see that $J$ is continuous and does not change sign on $(0,1)^{2}$.

Let $X, Y$ be absolutely continuous and independent random variables with range in $(0,1)$. Let us denote the densities by $f_{X}, f_{Y}$ respectively. Then, by the Transformation Theorem, the random variable

$$
(U, V)=\psi(X, Y)=\left(\frac{1-Y}{1-X Y}, 1-X Y\right)
$$

is absolutely continuous with density function $g$ defined by

$$
g(u, v):=f_{X}\left(\frac{1-v}{1-u v}\right) f_{Y}(1-u v) \frac{v}{1-u v}
$$

for all $(u, v) \in(0,1)^{2}$.

Wesołowski mentioned that if the random variables $X$ and $Y$ have beta distribution with density functions

$$
f_{X}(x)=\beta_{p, q}(x) \quad \text { and } \quad f_{Y}(x)=\beta_{p+q, r}(x), \quad x \in(0,1),
$$

respectively, then, setting these density functions equal to the right-hand side of (2), one finds out easily that the left-hand side of (2) can be factored into a function of $u$ and a function of $v$, both functions are beta densities with parameters $r, q$ and $r+q, p$, respectively.

Wesolowski asked a question about the converse of this observation: Assume that $X$ and $Y$ are independent and the random vector $(U, V)=\psi(X, Y)$ has independent components. Is it true in this case that $X, Y, U$ and $V$ have beta distribution?

This question has been answered in the affirmative by Wesołowski, assuming that $X, Y, U$ and $V$ have strictly positive and locally integrable densities on $(0,1)$.

If $U$ and $V$ are independent with density functions $f_{U}, f_{V}$ respectively, then Wesołowski gets from (2) the functional equation

$$
f_{U}(u) f_{V}(v)=f_{X}\left(\frac{1-v}{1-u v}\right) f_{Y}(1-u v) \frac{v}{1-u v}, \quad(u, v) \in(0,1)^{2}
$$

for unknown density functions $f_{X}, f_{Y}, f_{U}, f_{V}:(0,1) \rightarrow \mathbb{R}_{+}$. In fact, since density functions are not uniquely determined (the density functions of a random variable may differ on a set of measure zero), the independence of $U$ and $V$ yields that (3) is valid only for almost every $(u, v) \in(0,1)^{2}$.

He determined the solution of (3) under the assumptions that the density functions are strictly positive and locally integrable on $(0,1)$.

The investigations of Wesołowski are based on the locally integrable real solutions $g_{1}, g_{2}, \alpha_{1}, \alpha_{2}:(0,1) \rightarrow \mathbb{R}$ of the following general functional equation

$$
g_{1}\left(\frac{1-x}{1-x y}\right)+g_{2}\left(\frac{1-y}{1-x y}\right)=\alpha_{1}(x)+\alpha_{2}(y) \quad(x, y \in(0,1)) .
$$

He asked the measurable solution of (3) and the general solution of (4) too. 
The main aims of this paper are

(I) to give the general solution of (3) for functions $f_{X}, f_{Y}, f_{U}, f_{V}:(0,1) \rightarrow \mathbb{R}_{+}$, as well as the general solution of (4) without any regularity conditions,

(II) to determine the solution of (3) under the following more natural assumptions:

- the density functions are measurable,

- (3) is satisfied for almost every $(u, v) \in(0,1)^{2}$.

\section{The General solution of (3)}

To determine the general solution of (3) (and later the general solution of (4)) we need the following general result of Gy. Maksa (see [7]) in connection with the generalized fundamental equation of information with four unknown functions.

Theorem 2. Let

$$
D_{0}=\left\{(x, y) \in \mathbb{R}^{2} \mid x, y, x+y \in(0,1)\right\} .
$$

Functions $F, G, H, K:(0,1) \rightarrow \mathbb{R}$ satisfy the functional equation

$$
F(x)+G\left(\frac{y}{1-x}\right)=H(y)+K\left(\frac{x}{1-y}\right) \quad\left((x, y) \in D_{0}\right),
$$

if and only if

$$
\begin{aligned}
& F(x)=l_{1}(1-x)+l_{2}(x)+a_{1} \quad(x \in(0,1)), \\
& G(x)=l_{1}(1-x)+l_{3}(x)-l_{3}(1-x)-a_{1}-b_{2} \quad(x \in(0,1)), \\
& H(x)=l_{1}(1-x)+l_{2}(1-x)+l_{3}(x)-l_{3}(1-x)+b_{1} \quad(x \in(0,1)), \\
& K(x)=l_{1}(1-x)+l_{2}(x)-l_{3}(1-x)+b_{2} \quad(x \in(0,1)),
\end{aligned}
$$

where $l_{i}: \mathbb{R}_{+} \rightarrow \mathbb{R}(i=1,2,3)$ satisfies the Cauchy logarithmic equation

$$
l_{i}(x y)=l_{i}(x)+l_{i}(y) \quad\left(x, y \in \mathbb{R}_{+}\right)
$$

and $a_{1}, b_{1}, b_{2} \in \mathbb{R}$ are arbitrary constants.

Lemma 1. If the functions $f_{X}, f_{Y}, f_{U}, f_{V}:(0,1) \rightarrow \mathbb{R}_{+}$satisfy (3) then

$$
\begin{aligned}
& f_{X}(x)=\exp \left[l_{1}(x)+l_{2}(1-x)+a_{1}\right] \quad(x \in(0,1)), \\
& \frac{x f_{U}(x)}{f_{V}(x)}=\exp \left[-l_{1}(1-x)-l_{2}(x)+l_{2}(1-x)-b_{1}\right] \quad(x \in(0,1)),
\end{aligned}
$$

where $l_{i}: \mathbb{R}_{+} \rightarrow \mathbb{R}(i=1,2)$ satisfies (6) and $a_{1}, b_{1} \in \mathbb{R}$ are arbitrary constants.

Proof. Equation (3) can be written in the form

$$
u f_{U}(u) f_{V}(v)=f_{X}\left(\frac{1-v}{1-u v}\right) \frac{u v}{1-u v} f_{Y}(1-u v) \quad(u, v \in(0,1)) .
$$

Since

$$
u, \quad \frac{u v}{1-u v}, \quad f_{U}(u), \quad f_{V}(v), \quad f_{X}\left(\frac{1-v}{1-u v}\right) \text { and } f_{Y}(1-u v)
$$

are positive for all $u, v \in(0,1)$, taking the logarithm of (9), we get that the functions $G_{1}, G_{2}, F_{1}, F_{2}:(0,1) \rightarrow \mathbb{R}$ defined by

$$
\begin{gathered}
G_{1}(u)=\ln \left[u f_{U}(u)\right], \quad G_{2}(u)=\ln \left[f_{V}(u)\right], \\
F_{1}(u)=\ln \left[f_{X}(u)\right], \quad F_{2}(u)=\ln \left[\frac{u}{1-u} f_{Y}(1-u)\right],
\end{gathered}
$$


satisfy the functional equation

$$
G_{1}(u)+G_{2}(v)=F_{1}\left(\frac{1-v}{1-u v}\right)+F_{2}(u v) \quad(u, v \in(0,1)) .
$$

Interchanging $u$ and $v$ in (11), we get

$$
G_{1}(v)+G_{2}(u)=F_{1}\left(\frac{1-u}{1-u v}\right)+F_{2}(u v) \quad(u, v \in(0,1)) .
$$

Subtracting this equation from (11), we obtain

$$
\left(G_{1}-G_{2}\right)(u)-\left(G_{1}-G_{2}\right)(v)=F_{1}\left(\frac{1-v}{1-u v}\right)-F_{1}\left(\frac{1-u}{1-u v}\right) \quad(u, v \in(0,1)) .
$$

Now insert in this equation $\frac{1-u}{1-u v}=x$ and $\frac{1-v}{1-u v}=y$, then $u=\frac{1-x}{y}, v=\frac{1-y}{x}$, $x, y \in(0,1), x+y>1$ and the functions $G=G_{2}-G_{1}, F_{1}$ satisfy the equation

$$
F_{1}(x)+G\left(\frac{1-y}{x}\right)=F_{1}(y)+G\left(\frac{1-x}{y}\right) \quad(x, y \in(0,1), x+y>1) .
$$

By the substitutions $x \rightarrow 1-x, y \rightarrow 1-y$, we get from this last equation that

$$
F_{1}(1-x)+G\left(\frac{y}{1-x}\right)=F_{1}(1-y)+G\left(\frac{x}{1-y}\right) \quad(x, y, x+y \in(0,1)),
$$

i.e., the functions $F:(0,1) \rightarrow \mathbb{R}, F(x)=F_{1}(1-x)$ and $G:(0,1) \rightarrow \mathbb{R}$ satisfy the functional equation

$$
F(x)+G\left(\frac{y}{1-x}\right)=F(y)+G\left(\frac{x}{1-y}\right) \quad(x, y, x+y \in(0,1)) .
$$

Equation (12) is a special case of equation (5) with $H=F, K=G$.

Thus, we get from the above mentioned theorem of Maksa that

$$
\begin{aligned}
& F(x)=l_{1}(1-x)+l_{2}(x)+a_{1} \quad(x \in(0,1)), \\
& G(x)=l_{1}(1-x)+l_{2}(x)-l_{2}(1-x)+b_{1} \quad(x \in(0,1)),
\end{aligned}
$$

where the function $l_{i}: \mathbb{R}_{+} \rightarrow \mathbb{R}(i=1,2)$ satisfies the functional equation (6) and $a_{1}, b_{1} \in \mathbb{R}$ are arbitrary constants.

Finally, using the definition $F_{1}, F$ and $G$, we infer the statement of Lemma 1 and so $(7)$ and $(8)$ for $f_{X}(x)$ and $\frac{x f_{U}(x)}{f_{V}(x)}$ respectively.

On the other hand, by the substitutions

$$
\frac{1-v}{1-u v}=x, \quad 1-u v=y \quad \Longleftrightarrow \quad u=\frac{1-y}{1-x y}, \quad v=1-x y,
$$

we get from (3) the equation

$$
x f_{X}(x) f_{Y}(y)=f_{U}\left(\frac{1-y}{1-x y}\right) \frac{x y}{1-x y} f_{V}(1-x y) \quad(x, y \in(0,1)) .
$$

Thus, similarly to Lemma 1 , we get the following result.

Lemma 2. If the functions $f_{X}, f_{Y}, f_{U}, f_{V}:(0,1) \rightarrow \mathbb{R}_{+}$satisfy (3) (and so (13)), then

$$
\begin{aligned}
& f_{U}(x)=\exp \left[l_{3}(x)+l_{4}(1-x)+a_{2}\right] \quad(x \in(0,1)), \\
& \frac{x f_{X}(x)}{f_{Y}(x)}=\exp \left[-l_{3}(1-x)-l_{4}(x)+l_{4}(1-x)-b_{2}\right] \quad(x \in(0,1)),
\end{aligned}
$$


where the function $l_{i}: \mathbb{R}_{+} \rightarrow \mathbb{R}(i=3,4)$ satisfies the functional equation (6) and $a_{2}, b_{2} \in \mathbb{R}$ are arbitrary constants.

Now we can formulate the main result of this part.

Theorem 3. Functions $f_{X}, f_{Y}, f_{U}, f_{V}:(0,1) \rightarrow \mathbb{R}_{+}$satisfy the functional equation (3) if and only if

$$
\begin{aligned}
& f_{X}(x)=\exp \left[l_{1}(x)+l_{2}(1-x)+a_{1}\right] \quad(x \in(0,1)), \\
& f_{Y}(x)=x \exp \left[l_{1}(x)+l_{2}(x)+l_{3}(1-x)+a_{1}+b_{2}\right] \quad(x \in(0,1)), \\
& f_{U}(x)=\exp \left[l_{2}(1-x)+l_{3}(x)+a_{2}\right] \quad(x \in(0,1)), \\
& f_{V}(x)=x \exp \left[l_{1}(1-x)+l_{2}(x)+l_{3}(x)+a_{2}+b_{1}\right] \quad(x \in(0,1)),
\end{aligned}
$$

where function $l_{i}(i=1,2,3)$ satisfies the Cauchy logarithmic equation (6) and $a_{1}, a_{2}, b_{1}, b_{2} \in \mathbb{R}$ are arbitrary constants.

Proof. Using formulae in (7), (8) in Lemma 1 and (14), (15) in Lemma 2, we get immediately (16), and that

$$
\begin{array}{r}
f_{Y}(x)=x \exp \left[l_{1}(x)+l_{2}(1-x)+l_{3}(1-x)+l_{4}(x)-l_{4}(1-x)+a_{1}+b_{2}\right] \\
f_{U}(x)=\exp \left[l_{3}(x)+l_{4}(1-x)+a_{2}\right] \\
f_{V}(x)=x \exp \left[l_{3}(x)+l_{4}(1-x)+l_{1}(1-x)+l_{2}(x)-l_{2}(1-x)+a_{2}+b_{1}\right]
\end{array}
$$

for all $x \in(0,1)$.

On the other hand, a simple calculation gives that functions (16), (20), (21) and (22) satisfy (3) iff

$$
l_{4}\left[\frac{(1-u)(1-v) u v}{1-u v}\right]=l_{2}\left[\frac{(1-u)(1-v) u v}{1-u v}\right] \quad(u, v \in(0,1)) .
$$

It is easy to see that the range of the function $h:(0,1)^{2} \rightarrow \mathbb{R}, h(u, v)=\frac{(1-u)(1-v) u v}{1-u v}$ contains the open interval $\left(0, \frac{1}{6}\right)$, thus $l_{4}(t)=l_{2}(t)$ if $t \in\left(0, \frac{1}{6}\right)$, i.e. $\left(l_{4}-l_{2}\right)(t)=0$ if $t \in\left(0, \frac{1}{6}\right)$.

The function $l_{4}-l_{2}: \mathbb{R}_{+} \rightarrow \mathbb{R}$ satisfies the Cauchy logarithmic equation (6), too. Thus (see [2], [3]) $l_{4}-l_{2} \equiv 0$. This implies that functions (16), (20), (21), (22) satisfy (3) if and only if $l_{4} \equiv l_{2}$, which implies the statement of our Theorem 3.

From Theorem 3, we can easily obtain

Corollary 1. The continuous (or measurable) functions $f_{X}, f_{Y}, f_{U}, f_{V}:(0,1) \rightarrow$ $\mathbb{R}_{+}$satisfy the functional equation (3) iff

$$
\begin{aligned}
& f_{X}(x)=e^{a_{1}} x^{p-1}(1-x)^{q-1} \quad(x \in(0,1)) \\
& f_{Y}(x)=e^{a_{1}+b_{2}} x^{p+q-1}(1-x)^{r-1} \quad(x \in(0,1)), \\
& f_{U}(x)=e^{a_{2}} x^{r-1}(1-x)^{q-1} \quad(x \in(0,1)) \\
& f_{V}(x)=e^{a_{2}+b_{1}} x^{q+r-1}(1-x)^{p-1} \quad(x \in(0,1)),
\end{aligned}
$$

where $a_{1}, a_{2}, b_{1}, b_{2}, p, q, r \in \mathbb{R}$ are arbitrary constants. 
Proof. By Theorem 3, the functions $f_{X}, f_{Y}, f_{U}, f_{V}:(0,1) \rightarrow \mathbb{R}_{+}$satisfy (3) iff they are of the forms $(16),(17),(18),(19)$, which implies easily that

$$
\begin{aligned}
& l_{2}(x)=\log \left[\frac{(1-x) f_{X}(x) f_{U}(1-x)}{f_{V}(1-x)}\right]+b_{1}-a_{1} \quad(x \in(0,1)), \\
& l_{1}(x)=\log \left[f_{X}(x)\right]-l_{2}(1-x)-a_{1} \quad(x \in(0,1)) \\
& l_{3}(x)=\log \left[f_{V}(x)\right]-l_{1}(1-x)-l_{2}(x)-\log x-a_{2}-b_{1} \quad(x \in(0,1)) .
\end{aligned}
$$

By the continuity (or measurability) of functions $f_{X}, f_{U}, f_{V},(27)$ implies that $l_{2}$ is continuous (or measurable) on $(0,1)$. Now, by the continuity (or measurability) of $l_{2}$ and $f_{X}$, we get from (28) that $l_{1}$ is continuous (or measurable) on $(0,1)$, too. Finally (29), using the continuity (or measurability) of $l_{1}, l_{2}$ and $f_{V}$, implies the continuity (or measurability) of $l_{3}$.

$l_{1}, l_{2}, l_{3}: \mathbb{R}_{+} \rightarrow \mathbb{R}$ satisfy the Cauchy logarithmic equation (6) and are continuous (or measurable) on $(0,1)$. These imply (see [2], [3]) that

$$
l_{1}(x)=A_{1} \log x, \quad l_{2}(x)=A_{2} \log x, \quad l_{3}(x)=A_{3} \log x \quad\left(x \in \mathbb{R}_{+}\right),
$$

where $A_{i} \in \mathbb{R} \quad(i=1,2,3)$ is arbitrary constant.

Setting this form of $l_{i}(i=1,2,3)$ into (16), (17), (18), (19), an easy calculation shows that

$$
\begin{aligned}
& f_{X}(x)=e^{a_{1}} x^{A_{1}}(1-x)^{A_{2}} \quad(x \in(0,1)), \\
& f_{Y}(x)=e^{a_{1}+b_{2}} x^{A_{1}+A_{2}+1}(1-x)^{A_{3}} \quad(x \in(0,1)), \\
& f_{U}(x)=e^{a_{2}} x^{A_{3}}(1-x)^{A_{2}} \quad(x \in(0,1)), \\
& f_{V}(x)=e^{a_{2}+b_{1}} x^{A_{2}+A_{3}+1}(1-x)^{A_{1}} \quad(x \in(0,1)) .
\end{aligned}
$$

These imply, with constants $p=A_{1}+1, q=A_{2}+1$ and $r=A_{3}+1$, the statement of Corollary 1.

Remark 1. Functions $f_{X}, f_{Y}, f_{U}, f_{V}:(0,1) \rightarrow \mathbb{R}_{+}$, such that logarithms of these functions are locally integrable, satisfy (3) iff they are of the forms (23), (24), (25), (26), where $a_{1}, a_{2}, b_{1} \in \mathbb{R}$ and $p, q, r \in \mathbb{R}_{+}$are arbitrary constants.

\section{The measurable solution of (3) Satisfied almost everywhere}

Here we need the following result of A. Járai (see [5] and [6]).

Theorem 4 (Járai). Let $Z$ be a regular space, $Z_{i}(i=1,2, \ldots, n)$ topological spaces and $T$ a first countable topological space. Let $Y$ be an open subset of $\mathbb{R}^{k}, X_{i}$ an open subset of $\mathbb{R}^{r_{i}} \quad(i=1,2, \ldots, n)$ and $D$ an open subset of $T \times Y$. Let $T^{\prime} \subset T$ be a dense subset, $f: T^{\prime} \rightarrow Z, \quad g_{i}: D \rightarrow X_{i}$ and $h: D \times Z_{1} \times \ldots \times Z_{n} \rightarrow Z$. Suppose that the function $f_{i}$ is almost everywhere defined on $X_{i}$ with values in $Z_{i}$ $(i=1,2, \ldots n)$ and the following conditions are satisfied:

(1) for all $t \in T^{\prime}$ for almost all $y \in D_{t}$

$$
f(t)=h\left(t, y, f_{1}\left(g_{1}(t, y)\right), \ldots, f_{n}\left(g_{n}(t, y)\right)\right),
$$

where $D_{t}=\{y \in Y:(t, y) \in D\}$

(2) for each fixed $y$ in $Y$, the function $h$ is continuous in the other variables;

(3) $f_{i}$ is $\lambda^{r_{i}}$ measurable, i.e. $f_{i}$ is Lebesgue measurable on $\mathbb{R}^{r_{i}},(i=1,2, \ldots, n)$;

(4) $g_{i}$ and the partial derivative $\frac{\partial g_{i}}{\partial y}$ is continuous on $D(i=1,2, \ldots, n)$; 
(5) for each $t \in T$ there exist a $y$ such that $(t, y) \in D$ and the partial derivative $\frac{\partial g_{i}}{\partial y}$ has the rank $r_{i}$ at $(t, y) \in D \quad(i=1,2, \ldots, n)$.

Then there exists a unique continuous function $\tilde{f}$ such that $f=\tilde{f}$ almost everywhere on $T$, and if $f$ is replaced by $\tilde{f}$ then equation (35) is satisfied almost everywhere on $D$.

Lemma 3. If the measurable functions $f_{X}, f_{Y}, f_{U}, f_{V}:(0,1) \rightarrow \mathbb{R}_{+}$, satisfy equation (3) for almost all $(u, v) \in(0,1)^{2}$, then there exist unique continuous functions $\widetilde{f}_{X}, \widetilde{f}_{Y}, \widetilde{f}_{U}, \widetilde{f}_{V}:(0,1) \rightarrow \mathbb{R}_{+}$, such that $\widetilde{f}_{X}=f_{X}, \widetilde{f}_{Y}=f_{Y} \widetilde{f}_{U}=f_{U}, \widetilde{f}_{V}=f_{V}$ almost everywhere, and if $f_{X}, f_{Y}, f_{U}, f_{V}$ are replaced by $\widetilde{f}_{X}, \widetilde{f}_{Y}, \widetilde{f}_{U}, \widetilde{f}_{V}$ respectively, then equation (3) is satisfied everywhere on $(0,1)^{2}$.

Proof. First we prove that there exist unique continuous function $\widetilde{f}_{X}$ which is almost everywhere equal to $f_{X}$ on $(0,1)$ and replacing $f_{X}$ by $\widetilde{f}_{X}$, equation $(3)$ is satisfied almost everywhere.

With the substitution

$$
t=\frac{1-v}{1-u v}, \quad y=v
$$

we get from (3) the equation

$$
f_{X}(t)=\frac{f_{U}\left(\frac{y+t-1}{y t}\right) \frac{f_{V}(y)}{y}}{\frac{f_{Y}\left(\frac{1-y}{t}\right)}{\frac{1-y}{t}}},
$$

which is satisfied for almost all $(t, y) \in D$, where

$$
D=\{(t, y) \mid t, y \in(0,1), t+y>1\} .
$$

By Fubini's Theorem it follows that there exists $T \subseteq(0,1)$ of full measure such that, for all $t \in T$ equation (36) is satisfied for almost every $y \in D_{t}$, where

$$
D_{t}=\{y \in(0,1) \mid(t, y) \in D\} .
$$

Let us define the functions $g_{1}, g_{2}, g_{3}, h$ in the following way:

$$
\begin{gathered}
g_{1}(t, y)=\frac{y+t-1}{y t}, \\
g_{2}(t, y)=y, \\
g_{3}(t, y)=\frac{1-y}{t}, \\
h\left(t, y, z_{1}, z_{2}, z_{3}\right)=\frac{z_{1} z_{2}}{z_{3}},
\end{gathered}
$$

and let us now apply Theorem of Járai to (36) with the following casting:

$$
\begin{gathered}
f_{X}(t)=f(t), \quad f_{U}(t)=f_{1}(t), \quad \frac{f_{V}(t)}{t}=f_{2}(t), \quad \frac{f_{Y}(t)}{t}=f_{3}(t), \\
Z=\mathbb{R}_{+}, \quad Z_{i}=\mathbb{R}_{+}, \quad T=(0,1), \quad Y=(0,1), \quad X_{i}=(0,1),(i=1,2,3) .
\end{gathered}
$$

Hence the first assumption in the Theorem of Járai with respect to (36) is satisfied.

In the case of a fixed $y$, the function $h$ is continuous in the other variables, so the second assumption holds too.

Because the functions in equation (36) are measurable, the third assumption is trivial. 
The functions $g_{i}$ are continuous, the partial derivatives

$$
D_{2} g_{1}(t, y)=\frac{1-t}{y^{2} t}, \quad D_{2} g_{2}(t, y)=1, \quad D_{2} g_{3}(t, y)=-\frac{1}{t}
$$

are also continuous, so the fourth assumption holds too.

For each $t \in(0,1)$ there exist a $y \in(0,1)$ such that $(t, y) \in D$ and the partial derivatives don't equal zero in $(t, y)$, so they have the rank 1 . Thus the last assumption is satisfied in the Theorem of Járai.

So we get, from Járai's Theorem that there exists a unique continuous function $\widetilde{f}_{X}$ which is almost everywhere equal to $f_{X}$ on $(0,1)$ and $\tilde{f}_{X}, f_{Y}, f_{U}, f_{V}$ satisfy equation (36) almost everywhere, which is equivalent to equation

$$
f_{U}(u) f_{V}(v)=\tilde{f}_{X}\left(\frac{1-v}{1-u v}\right) f_{Y}(1-u v) \frac{v}{1-u v}
$$

for almost all $(u, v) \in(0,1)^{2}$.

By a similar argument, we can prove the same for the function $f_{Y}$.

From equation (37) with the substitution $t=1-u v, y=v$ we get the equation

$$
f_{Y}(t)=\frac{f_{U}\left(\frac{1-t}{y}\right) \frac{f_{V}(y)}{y} t}{\widetilde{f}_{X}\left(\frac{1-y}{t}\right)},
$$

which, by Fubini's Theorem again, is satisfied for almost all $t \in(0,1)$ and for almost all $y \in D_{t}$.

With the casting

$$
\begin{gathered}
g_{1}(t, y)=\frac{1-t}{y}, \quad g_{2}(t, y)=y, \quad g_{3}(t, y)=\frac{1-y}{t}, \\
h\left(t, y, z_{1}, z_{2}, z_{3}\right)=\frac{z_{1} z_{2}}{z_{3}}
\end{gathered}
$$

use the Theorem of Járai for the equation (38). In this case, we can also see that the assumptions of the Theorem of Járai are fulfilled, hence there exists a unique continuous function $\tilde{f}_{Y}$ which is almost everywhere equal to $f_{Y}$ on $(0,1)$ and $\widetilde{f}_{X}, \widetilde{f}_{Y}, f_{U}, f_{V}$ satisfy equation (38) almost everywhere, i.e.

$$
\widetilde{f}_{Y}(t)=\frac{f_{U}\left(\frac{1-t}{y}\right) \frac{f_{V}(y)}{y} t}{\widetilde{f}_{X}\left(\frac{1-y}{t}\right)},
$$

almost everywhere on $(0,1)^{2}$, which is equivalent to (3) replacing $f_{X}$ and $f_{Y}$ by $\widetilde{f}_{X}$ and $\widetilde{f}_{Y}$, i.e.

$$
f_{U}(u) f_{V}(v)=\tilde{f}_{X}\left(\frac{1-v}{1-u v}\right) \tilde{f}_{Y}(1-u v) \frac{v}{1-u v}
$$

for almost all $(u, v) \in(0,1)^{2}$.

Since $\psi=\psi^{-1}$, we get from (39) the equation

$$
\widetilde{f}_{X}(x) \widetilde{f}_{Y}(y)=f_{U}\left(\frac{1-y}{1-x y}\right) f_{V}(1-x y) \frac{y}{1-x y}
$$

for almost all $(x, y) \in(0,1)^{2}$. Equation (40) is dual to (39) by simple changing $\left(f_{U}, f_{V}\right)$ into $\left(f_{X}, f_{Y}\right)$.

By the same steps as in the case of $f_{X}$ and $f_{Y}$, we can prove that there exist unique continuous functions $\widetilde{f}_{U}$ and $\widetilde{f}_{V}$ which are almost everywhere equal to $f_{U}$ 
and $f_{V}$ on $(0,1)$, respectively, and replacing $f_{U}$ and $f_{V}$ by $\widetilde{f}_{U}$ and $\widetilde{f}_{V}$, respectively, the functional equation (40) and so the functional equation

$$
\widetilde{f}_{U}(u) \widetilde{f}_{V}(v)=\widetilde{f}_{X}\left(\frac{1-v}{1-u v}\right) \widetilde{f}_{Y}(1-u v) \frac{v}{1-u v}
$$

is satisfied almost everywhere in $(0,1)^{2}$, and hence on a dense set in $(0,1)^{2}$.

Then, by the continuity of functions involved in (41), it follows evidently that (41) is satisfied for all $(u, v) \in(0,1)^{2}$. Furthermore, $f_{X}=\widetilde{f}_{X}, f_{Y}=\widetilde{f}_{Y}, f_{U}=\widetilde{f}_{U}$ and $f_{V}=\widetilde{f}_{V}$ almost everywhere on $(0,1)$.

Now, using Lemma 3 and Corollary 1, one can easily prove the following

Theorem 5. The measurable functions $f_{X}, f_{Y}, f_{U}, f_{V}:(0,1) \rightarrow \mathbb{R}_{+}$satisfy the functional equation (3) for almost all $(u, v) \in(0,1)^{2}$ iff there exist positive constants $p, q, r, \varepsilon_{i}(i=1,2,3,4)$ with $\varepsilon_{1} \varepsilon_{4}=\varepsilon_{2} \varepsilon_{3}$ such that

$$
\begin{aligned}
& f_{X}(x)=\varepsilon_{1} x^{p-1}(1-x)^{q-1} \quad(x \in(0,1) \text { a.e. }), \\
& f_{Y}(y)=\varepsilon_{2} y^{p+q-1}(1-y)^{r-1} \quad(y \in(0,1) \text { a.e. }), \\
& f_{U}(u)=\varepsilon_{3} u^{r-1}(1-u)^{q-1} \quad(u \in(0,1) \text { a.e. }), \\
& f_{V}(v)=\varepsilon_{4} v^{q+r-1}(1-v)^{p-1} \quad(v \in(0,1) \text { a.e. }) .
\end{aligned}
$$

(Consequently $f_{X}, f_{Y}, f_{U}, f_{V}$ are density functions of beta distribution.)

Proof. Under the assumptions of our theorem, it follows from Lemma 3 that there exist unique continuous functions $\widetilde{f}_{X}, \widetilde{f}_{Y}, \widetilde{f}_{U}, \widetilde{f}_{V}:(0,1) \rightarrow \mathbb{R}_{+}$such that $f_{X}=\widetilde{f}_{X}$, $f_{Y}=\widetilde{f}_{Y}, f_{U}=\widetilde{f}_{U}, f_{V}=\widetilde{f}_{V}$ almost everywhere and functional equation (41) is satisfied for all $(u, v) \in(0,1)^{2}$. Then we infer from Corollary 1 that continuous functions $\widetilde{f}_{X}, \widetilde{f}_{Y}, \widetilde{f}_{U}, \widetilde{f}_{V}:(0,1) \rightarrow \mathbb{R}_{+}$satisfy (41) iff they are of the form (23), (24), (25) and (26) respectively. Summarizing these, we have the statement of our theorem with constants $\varepsilon_{1}=e^{a_{1}}, \varepsilon_{2}=e^{a_{1}+b_{2}}, \varepsilon_{3}=e^{a_{2}}, \varepsilon_{4}=e^{a_{2}+b_{1}}$.

Corollary 2. If $X$ and $Y$ are absolutely continuous and independent random variables (and the support of $X$ and $Y$ are equal $(0,1))$ such that the random variables, defined by

$$
U=\frac{1-Y}{1-X Y}, \quad V=1-X Y,
$$

are also independent, then $X, Y, U$ and $V$ belong to the family of beta distributions. That is, with the notations of the previous theorem, $X, Y, U$ and $V$ have beta distributions with parameters $p, q ; p+q, r ; r, q$ and $q+r, p$, respectively.

Remark 2. The following problem is still open (Referee's suggestion): Is it possible to solve (3) holding almost everywhere for unknown functions assuming values in $[0, \infty)$ ?

\section{The General solution of (4)}

By the substitutions

$$
\frac{1-x}{1-x y}=u, \quad \frac{1-y}{1-x y}=v
$$


and consequently

we get from

$$
x=\frac{1-u}{v}, \quad y=\frac{1-v}{u}, \quad u, v \in(0,1), \quad u+v>1,
$$

$$
g_{1}\left(\frac{1-x}{1-x y}\right)+g_{2}\left(\frac{1-y}{1-x y}\right)=\alpha_{1}(x)+\alpha_{2}(y) \quad(x, y \in(0,1))
$$

the functional equation

$$
g_{1}(u)+g_{2}(v)=\alpha_{1}\left(\frac{1-u}{v}\right)+\alpha_{2}\left(\frac{1-v}{u}\right) \quad(u, v \in(0,1), u+v>1) .
$$

Replacing $u$ by $1-x$ and $v$ by $1-y$ in (42), we get

$$
g_{1}(1-x)+g_{2}(1-y)=\alpha_{1}\left(\frac{x}{1-y}\right)+\alpha_{2}\left(\frac{y}{1-x}\right) \quad(x, y, x+y<1) .
$$

This implies that the functions $F, G, H, K:(0,1) \rightarrow \mathbb{R}$ defined by

$$
F(x)=g_{1}(1-x), G(x)=-\alpha_{2}(x), H(x)=-g_{2}(1-x), K(x)=\alpha_{1}(x)
$$

satisfy the functional equation (5).

Thus, theorem of Maksa implies that

$$
\begin{array}{cl}
g_{1}(1-x)= & l_{1}(1-x)+l_{2}(x)+a_{1}, \\
-g_{2}(1-x)= & l_{1}(1-x)+l_{2}(1-x)+l_{3}(x)-l_{3}(1-x)+b_{1}, \\
\alpha_{1}(x)= & l_{1}(1-x)+l_{2}(x)-l_{3}(1-x)+b_{2}, \\
-\alpha_{2}(x)= & l_{1}(1-x)+l_{3}(x)-l_{3}(1-x)+b_{1}-a_{1}+b_{2}
\end{array}
$$

for all $x \in(0,1)$. Finally from (43) we get the following result.

Theorem 6. The functions $g_{1}, g_{2}, \alpha_{1}, \alpha_{2}:(0,1) \rightarrow \mathbb{R}$ satisfy the functional equation (4) if and only if

$$
\begin{aligned}
& g_{1}(x)=A(x)+B(1-x)+a_{1} \quad(x \in(0,1)), \\
& g_{2}(x)=C(x)+D(1-x)-b_{1} \quad(x \in(0,1)), \\
& \alpha_{1}(x)=A(1-x)+B(x)+D(1-x)+b_{2} \quad(x \in(0,1)), \\
& \alpha_{2}(x)=B(1-x)+C(1-x)+D(x)-b_{1}+a_{1}-b_{2} \quad(x \in(0,1))
\end{aligned}
$$

where functions $A, B, C, D: \mathbb{R}_{+} \rightarrow \mathbb{R}$ satisfy the logarithmic Cauchy equation (6), $A+B+C+D=0$ and $a_{1}, b_{1}, b_{2} \in \mathbb{R}$ are arbitrary constants.

Proof. From (43) with notations $A=l_{1}, B=l_{2}, D=-l_{3}$ and $l_{3}-l_{1}-l_{2}=C$ we get (44). Functions $A, B, C, D: \mathbb{R}_{+} \rightarrow \mathbb{R}$ satisfy (6).

An easy calculation shows that the functions $g_{1}, g_{2}, \alpha_{1}, \alpha_{2}$, defined by (44) satisfy (4) indeed, if $A+B+C+D=0$.

Corollary 3. The measurable functions $g_{1}, g_{2}, \alpha_{1}, \alpha_{2}:(0,1) \rightarrow \mathbb{R}$ satisfy the functional equation (4) iff

$$
\begin{aligned}
& g_{1}(x)=\alpha \log x+\beta \log (1-x)+a_{1}, \\
& g_{2}(x)=\gamma \log x+\delta \log (1-x)-b_{1}, \\
& \alpha_{1}(x)=\beta \log x-(\beta+\gamma) \log (1-x)+b_{2}, \\
& \alpha_{2}(x)=(\beta+\gamma) \log (1-x)+\delta \log x-b_{1}+a_{1}-b_{2},
\end{aligned}
$$

where $\alpha, \beta, \gamma, \delta, a_{1}, b_{1}, b_{2} \in \mathbb{R}$ are arbitrary constants with $\alpha+\beta+\gamma+\delta=0$. 
Proof. By Theorem 6, functions $g_{1}, g_{2}, \alpha_{1}, \alpha_{2}:(0,1) \rightarrow \mathbb{R}$ satisfy (4) iff the functions are of the form (44), which implies easily that

$$
\begin{aligned}
& A(x)=g_{1}(x)-\alpha_{2}(x)+g_{2}(1-x)-b_{2} \quad(x \in(0,1)), \\
& B(x)=g_{1}(1-x)-A(1-x)-a_{1} \quad(x \in(0,1)), \\
& C(x)=\alpha_{2}(1-x)-\alpha_{1}(x)+A(1-x)+b_{1}-a_{1}+2 b_{2} \quad(x \in(0,1)), \\
& D(x)=-A(x)-B(x)-C(x) \quad(x \in(0,1)) .
\end{aligned}
$$

The measurability of functions $g_{1}, g_{2}, \alpha_{1}, \alpha_{2}$ on $(0,1)$ imply, by these equalities, the measurability of functions $A, B, C$ and finally $D$ on $(0,1)$.

Furthermore, $A, B, C, D: \mathbb{R}_{+} \rightarrow \mathbb{R}$ satisfy the Cauchy logarithmic equation (6). These imply that

$$
A(x)=\alpha \log x, B(x)=\beta \log x, C(x)=\gamma \log x, D(x)=\delta \log x \quad x \in \mathbb{R}_{+}
$$

where $\alpha, \beta, \gamma, \delta \in \mathbb{R}$ are arbitrary constants with $\alpha+\beta+\gamma+\delta=0$. Setting (45) into (44), we get immediately the statement of our corollary.

\section{REFERENCES}

[1] Aczél, J., Lectures on Functional Equations and Their Applications, Academic Press, New York, 1966

[2] Aczél, J. and Dhombres, J., Functional Equations in Several Variables, Cambridge University Press, Cambridge, 1989.

[3] Kuczma, M., Introduction to the Theory of Functional Equations and Inequalities, Państwowe Wydawnictwo Naukowe - Uniwersytet Śląski, Warszawa-Kraków-Katowice, 1985.

[4] Giri, N. C., Introduction to probability and statistics, Marcel Dekker, New York, 1974.

[5] Járai, A., Measurable solutions of functional equations satisfied almost everywhere, Mathematica Pannonica 10/1 (1999), 103-110.

[6] Járai, A., Regularity Properties of Functional Equations in Several Variables, Springer, Adv. Math. Dordrecht, Vol. 8, 2005.

[7] Maksa, Gy., Solution on the open triangle of the generalized fundamental equation of information with four unknown functions, Utilitas Math. 21 (1982), 267-282.

[8] Wesołowski, J., On a functional equation related to an independence property for beta distributions, Aequationes Math., 66 (2003), 156-163.

Institute of Mathematics, University of Debrecen, H-4010 Debrecen, P. O. Box 12, HUNGARY

E-mail address: lajko@math.klte.hu

E-mail address: mefru@math.klte.hu 